\title{
How does the GINA definition of control correlate with quality of life and sputum cellularity?
}

\author{
Marcia Margaret Menezes Pizzichini $\mathbb{1}^{1}{ }^{2}$, Cristiane Cinara Rocha ${ }^{1}$, \\ Michelle Gonçalves de Souza Tavares ${ }^{1,2}$, Leila John Marques Steidle ${ }^{1}$, \\ Rosemeri Maureci da Silva1, Felipe dal Pizzol ${ }^{1}$, Peter G. Gibson ${ }^{3,4}$ and \\ Emilio Pizzichini ${ }^{1}$
}

Affiliations: ${ }^{1}$ NUPAIVA Research Center, Federal University of Santa Catarina, Florianópolis, Brazil. ${ }^{2}$ CAPES (Coordenação de Aperfeiçoamento de Pessoal de Nível Superior), Brazil. ${ }^{3}$ Centre of Excellence in Severe Asthma and Priority Research Centre for Healthy Lungs, Faculty of Health, University of Newcastle, Callaghan, Australia. ${ }^{4}$ Dept of Respiratory and Sleep Medicine, John Hunter Hospital, New Lambton Heights, Australia.

Correspondence: Marcia Margaret Menezes Pizzichini, Universidade Federal de Santa Catarina, UFSC NUPAIVA, Hospital Universitário, Campus Universitário, Trindade, 88040-970 Florianópolis, Santa Catarina, Brazil. E-mail: marcia.pizzichini6@agmail.com

ABSTRACT Since 2014, the Global Initiative for Asthma (GINA) has stated that asthma control should be measured using four questions concerning diurnal and nocturnal symptoms, activity limitation, and rescue medication use. We assessed how asthma control by this definition correlates with airway inflammation and quality of life.

113 asthmatic subjects consecutively recruited from their routine clinical appointment underwent spirometry, sputum induction and answered the Standardised Asthma Quality of Life Questionnaire (AQLQ(S)) during a single visit.

43 (38.1\%), 37 (32.7\%) and 33 (29.2\%) subjects had controlled asthma, partly controlled asthma and uncontrolled asthma, respectively. The majority of subjects with controlled asthma (67.4\%) had paucigranulocytic sputum. Eosinophilic sputum was present in all levels of asthma control. Although most subjects with controlled asthma (58.1\%) achieved an AQLQ(S) score $\geqslant 6$ (minimal or no impairment), the remaining patients $(41.9 \%)$ had moderate/some impairment (AQLQ(S) score $<6$ and $\geqslant 3$ ) due to activity impairment and environmental exposure.

The present GINA definition of current symptom control reflects control of airway inflammation. However, quality of life impairment can be present even in these patients. Measuring quality of life may provide useful information when evaluating asthma control.

@ERSpublications

Quality of life impairment can be present even in patients with controlled asthma and controlled airway inflammation http://ow.ly/RCmz30n4EUT

Cite this article as: Pizzichini MMM, Rocha CC, de Souza Tavares MGonç, et al. How does the GINA definition of control correlate with quality of life and sputum cellularity? ERJ Open Res 2019; 5: 00146-2018 [https://doi.org/10.1183/23120541.00146-2018].

This article has supplementary material available from openres.ersjournals.com

Received: Sept 032018 | Accepted after revision: Dec 032018

Copyright $\odot$ ERS 2019. This article is open access and distributed under the terms of the Creative Commons Attribution Non-Commercial Licence 4.0 


\section{Introduction}

The treatment and management of asthma with the aim of achieving and maintaining control is now well established [1,2]. To be beneficial in clinical practice, the assessment of asthma control should ideally be easily applicable and reliable, thereby allowing physicians and patients to make informed decisions at the point of care. However, asthma control is a multidimensional measure with features that are complementary to each other, including clinical, functional and disease activity [3]. Hence, a quick and easy assessment may not offer a comprehensive or precise estimation of asthma control.

The concept of asthma control is evolving, which is in line with recent evidence reflecting the heterogeneity of asthma and the various dimensions of disease activity. In this regard, the Global Initiative for Asthma (GINA) strategy since 2010, following the recommendations of an American Thoracic Society/European Respiratory Society task force, has suggested that measuring asthma control should include two domains: current clinical control and future risks [1]. In the 2014 revised version of the GINA strategy [4], the current limitations domain was replaced by "symptom control", which, as in many other asthma control questionnaires [5-7], includes daytime symptoms, nocturnal waking due to asthma, rescue medication needed for symptoms relief and any activity limitation due to asthma. The domain of future risks, now renamed "risk factors for poor outcomes", comprises predictors of adverse outcomes such as exacerbations, exposures, comorbidities, low forced expiratory volume in $1 \mathrm{~s}$ (FEV1) and blood or sputum eosinophilia, among others. In addition, the GINA documents $[2,8]$ emphasise that both control domains should be assessed periodically with particular attention paid to risk factors for exacerbations, fixed airflow limitations and side-effects from treatment. There is no mention, however, of measurements of quality of life.

The evaluation of quality of life and airway inflammation together with asthma control has been scarcely studied. In a previous report, Volbeda et al. [9] showed that the level of asthma control, based on a composite measure of clinical parameters used in the GOAL (Gaining Optimal Asthma ControL) study [10], is associated with inflammatory markers of eosinophilic inflammation, with little difference between totally controlled and well-controlled asthma. Although it seems obvious to assume that the better the asthma control is, the better the quality of life will be, it is unknown whether this assumption is true for the different levels of asthma control according to the GINA criteria for current symptom control [4].

\section{Methods}

\section{Study design}

This cross-sectional study involved one visit to the research clinic, where written informed consent was obtained and patients who fulfilled the inclusion criteria were enrolled in the study. Asthmatic outpatients were consecutively recruited from their routine clinical visits. Asthma control according to the GINA guidelines [4], quality of life, spirometry on treatment and induced sputum were simultaneously assessed.

\section{Subjects}

Adults aged 18-70 years with a diagnosis of asthma who visited the pulmonary outpatient clinics of one academic institution (Federal University of Santa Catarina, Florianópolis, Brazil) and one nonacademic institution (Respirar Centro de Medicina Respiratoria, Florianópolis, Brazil) were consecutively recruited and screened for possible participation in the study. All potential participants had been receiving asthma treatment for at least 1 year and were lifelong nonsmokers or ex-smokers (cessation for $>1$ year and smoking exposure $<10$ pack-years). The asthma was objectively confirmed by the demonstration of variable airflow limitation or of methacholine causing a $20 \%$ fall in FEV1 from baseline (PC20 methacholine $\left.<8 \mathrm{mg} \cdot \mathrm{mL}^{-1}\right)$. None of the participants had other lung diseases, a respiratory infection in the previous 1 month or a recent asthma exacerbation within the previous 6 months. The local medical ethics committee approved the study protocol and all subjects gave their written informed consent.

\section{Procedures}

Subject characteristics were documented using a structured questionnaire, control of asthma was assessed according to the GINA 2014 criteria [4] and quality of life was determined using the Standardised Asthma Quality of Life Questionnaire (AQLQ(S)) [11].

Allergy skin tests were performed by the modified skin prick technique [12] using 14 common allergen extracts. Spirometry was performed on treatment according to the American Thoracic Society standards [13] before and $10 \mathrm{~min}$ after salbutamol $200 \mu \mathrm{g}$ inhaled through an Aerochamber (Trudell Medical, London, ON, Canada). Reference values were obtained from CRAPO et al. [14]. Methacholine inhalation tests were carried out using the tidal breathing method [15].

The AQLQ(S) comprises 32 questions divided into four domains (symptoms, emotional function, environment stimuli and activity limitation). Responses to each question were graded on a seven-point scale, where 1 indicates maximum impairment and 7 indicates no impairment. The total score is obtained 
by averaging the responses to the 32 questions and the scores of the individual domains are obtained from the mean of the responses to the relevant questions. Quality of life was assessed using a cross-cultural adaptation for the Portuguese spoken in Brazil of the standard self-administered AQLQ(S) [16]. The $\mathrm{AQLQ}(\mathrm{S})$ minimum clinically important difference is $\geqslant 0.5$ units [17]. The magnitude of quality of life impairment was considered minimal or no impairment if the total score was $\geqslant 6$, moderate/some impairment if the total score was $\geqslant 3$ and $<6$, and severe impairment if the total score was $<3$ [18].

Sputum was induced and processed as previously described [19]. The sputum was labelled eosinophilic if sputum eosinophils were $\geqslant 3.0 \%$, neutrophilic when sputum neutrophils were $\geqslant 60.0 \%$ and paucigranulocytic if neither [20].

\section{Analysis}

Descriptive statistics were used to summarise the demographic characteristics of the patients. Continuous data were summarised by the arithmetic mean and standard deviation or $95 \%$ confidence interval. Variables with a skewed distribution (total cell count, percentage eosinophils and percentage lymphocytes) were $\log$ transformed before analysis. Differences between groups were evaluated by ANOVA. For comparisons of the AQLQ(S) score, differences between groups were evaluated by univariate analysis adjusted for age. The significance of differences between groups was determined in the post hoc analysis using Bonferroni's test. Differences between categorical variables were analysed by the Chi-squared test. The relationship between quality of life and sputum inflammatory cells was analysed using Pearson's correlation coefficient. All tests were two-tailed and significance was accepted at the level of $95 \%$. Statistical analysis was performed using SPSS for Windows version 22.0 (SPSS, Chicago, IL, USA).

\section{Results}

We recruited 130 consecutive subjects with asthma and 113 of these patients were eligible for inclusion in the study. Asthma was controlled in 43 (38.1\%) subjects, partly controlled in $37(32.7 \%)$ subjects and uncontrolled in 33 (29.2\%) subjects (table 1). Except by age, which was significantly lower in subjects with controlled asthma (mean 38.1 versus 46.2 (partly controlled asthma) versus 46.7 (uncontrolled asthma) years; $\mathrm{p}=0.01$ ), there were no differences between the clinical characteristics of the subjects from the three control groups. Functional characteristics, as expected, differed significantly between groups. The mean pre-bronchodilator FEV1 \% pred and the FEV1/forced vital capacity ratio were $90.0 \%$ (95\% CI 85.9-94.1\%) and 0.77 (95\% CI 0.74-0.80), respectively, in the controlled asthma group; $81.2 \%$ (95\% CI

\section{TABLE 1 Clinical and functional characteristics of subjects}

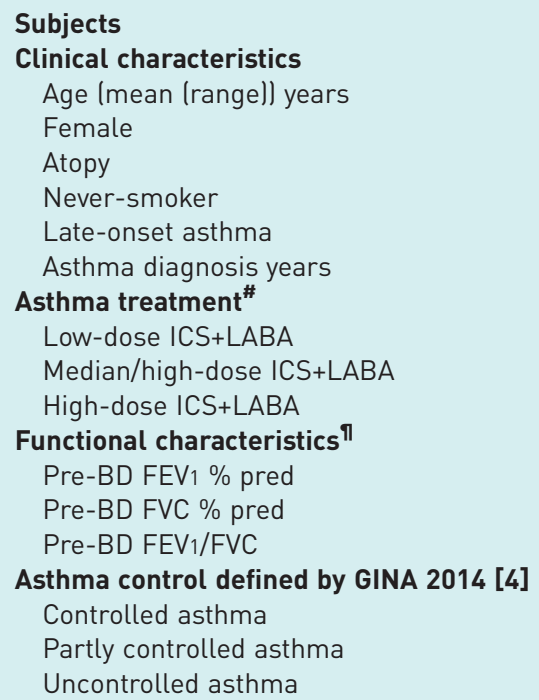

Data are presented as $n$, mean $(95 \%, \mathrm{Cl})$ or $n(\%)$, untess otherwise stated. Atopy means one or more

Data are presented as $\mathrm{n}$, mean $(95 \% \mathrm{CI})$ or $\mathrm{n}(\%)$, unless otherwise stated. Atopy means one or more positive allergy skin prick tests with a wheal $>2 \mathrm{~mm}$ more than negative controls. ICS: inhaled corticosteroid; LABA: long-acting $\mathrm{B}_{2}$-agonist; $\mathrm{BD}$ : bronchodilator (salbutamol); $\mathrm{FEV} 1$ : forced expiratory volume in $1 \mathrm{~s}$; FVC: forced vital capacity; GINA: Global Initiative for Asthma. " : low, medium/high and high doses of ICS according to GINA [2]; " : FEV1 and FVC measured on treatment (predicted values from CRAPO et al. [14]). 
75.8-86.7\%) and 0.70 (95\% CI 0.66-0.73), respectively, in the partly controlled asthma group; and $66.8 \%$ (95\% CI 60.9-72.8\%) and 0.63 (95\% CI 0.59-0.67), respectively, in the uncontrolled asthma group ( $\mathrm{p}<0.001$ for all between-group comparisons with uncontrolled asthma, and $\mathrm{p}<0.05$ for the comparison between controlled and partly controlled asthma).

\section{Asthma control, airway inflammation and quality of life}

The majority of subjects with controlled asthma (67.4\%) had paucigranulocytic sputum. Eosinophilic airway inflammation was present in $25.2 \%, 35.1 \%$ and $33.3 \%$ of the subjects with controlled, partly controlled and uncontrolled asthma, respectively (supplementary figure S1). Multiple linear regression analysis showed that asthma control, but not FEV1 or sputum eosinophilia (supplementary figure S2), was independently associated with quality of life $\left(R^{2}=0.510, p<0.001\right)$, explaining $50 \%$ of the variability in the AQLQ(S) score.

\section{Asthma control versus asthma-specific quality of life score}

The mean values for the overall AQLQ(S) score were significantly higher in subjects with controlled asthma $(6.1,95 \%$ CI 5.7-6.4) than in subjects with partly controlled asthma (5.3, 95\% CI 4.9-5.7) or those with uncontrolled asthma (3.6, 95\% CI 3.2-4.0) $(\mathrm{p}<0.002)$ (figure 1). The mean differences between groups for the overall AQLQ(S) scores were both clinically important and statistically significant for the comparisons between controlled asthma versus partly controlled asthma, controlled asthma versus uncontrolled asthma and partly controlled asthma versus uncontrolled asthma. The same results were

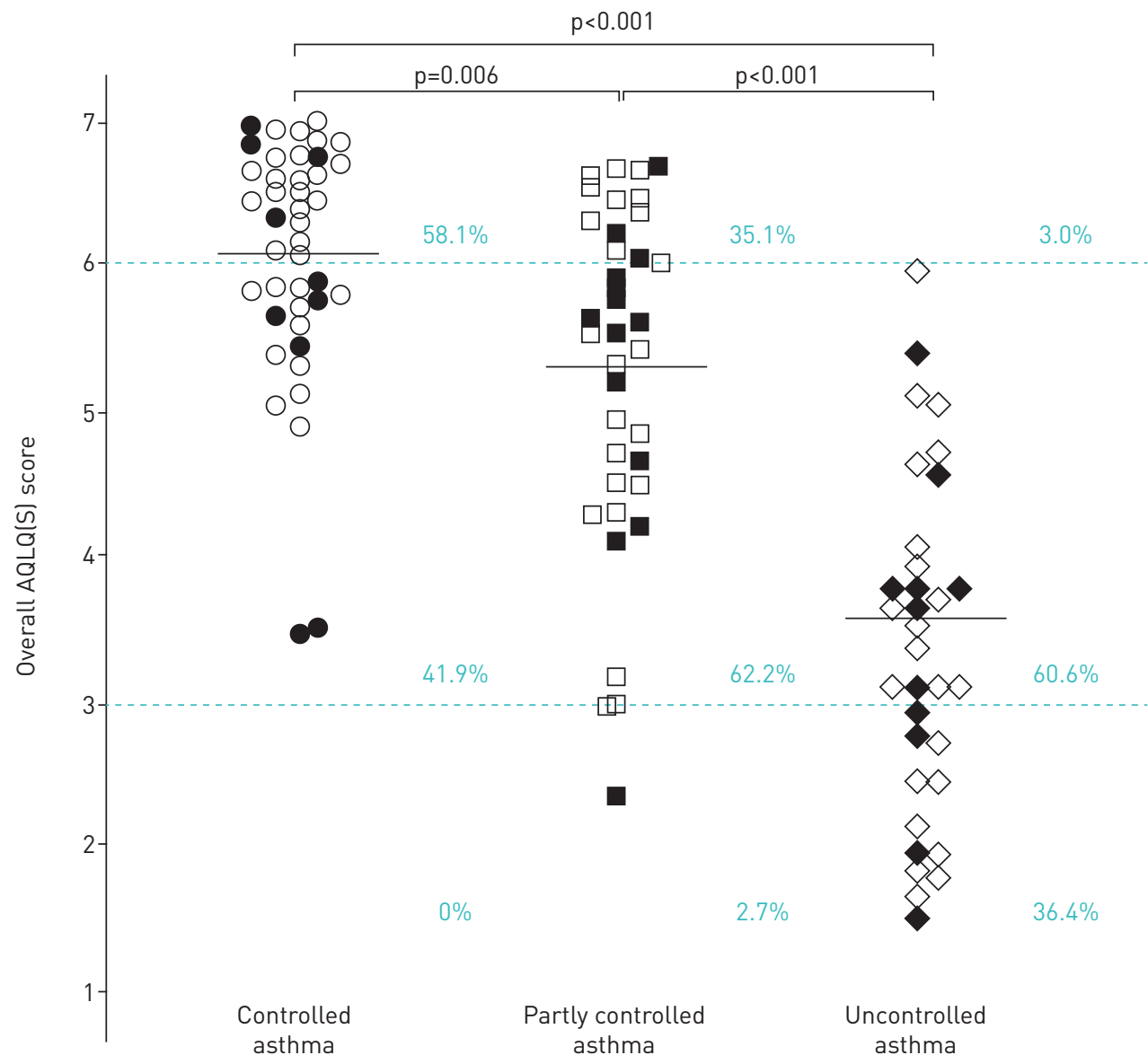

FIGURE 1 Individual plots of overall Standardised Asthma Quality of Life Questionnaire (AQLQ(S)) scores in each Global Initiative for Asthma level of asthma control (controlled, partly controlled and uncontrolled). Solid symbols: eosinophilic sputum; open symbols: noneosinophilic sputum. The mean of each group is indicated. The upper dashed line represents the lower limit of the AQLQ(S) score $(\geqslant 6)$ that indicates minimal or no impairment; the lower dashed line represents the upper limit of the AQLQ(S) score $(<3)$ that indicates severe impairment; the area between the dashed lines represents AQLQ(S) score $\geqslant 3$ and $<6$, indicating moderate/some impairment. Percentage values indicate the proportion of subjects in each level of asthma control with minimal or no impairment, moderate/some impairment, or severe impairment. 
observed for the AQLQ(S) comparison of symptoms, activity limitation and environmental stimuli domains (table 2 and figures 2-4).

The impact of asthma control on quality of life as measured by the magnitude of impairment was significantly different between the levels of control $(\mathrm{p}<0.001)$ (figure 1). Although most subjects $(58.1 \%)$ with controlled asthma achieved an AQLQ $(S)$ score $\geqslant 6$ (minimal or no impairment), the remaining patients $(41.9 \%)$ had moderate/some impairment (AQLQ(S) score $<6$ and $\geqslant 3$ ), which was due to impairments in activities (figure 2), environmental exposure (figure 3) and symptoms (figure 4). Additionally, some to moderate limitation of strenuous activities (e.g. hurrying, exercising, running up the stairs, sports) was reported by $40.6 \%$ of 64 asthmatic subjects who had previously reported not having any activity limitations in the previous 4 weeks. Asthma symptoms when exposed to smoking, air pollution or dust were reported by $20 \%$ of subjects who denied having asthma symptoms in the last 4 weeks. Some to moderate discomfort or distress caused by coughing was reported by $18.6 \%$ of subjects with controlled asthma.

\section{Discussion}

The results of the present study, which aimed to determine the relationships between quality of life and airway inflammation with asthma control as defined by the most recent GINA guidelines [2], showed three main findings. First, the majority of subjects with controlled asthma were found to have paucigranulocytic sputum, suggesting control of inflammation. Second, although the AQLQ(S) scores differed significantly between the three levels of asthma control, $\sim 40 \%$ of subjects with controlled asthma were found to have moderate/some impairment, and this impairment was chiefly due to activity limitations and environmental stimuli exposure. Third, symptom control, but not lung function or sputum eosinophilia, was shown to correlate with quality of life, explaining half of the variability in the AQLQ(S) score. These results indicate that, despite the good correlation between current asthma symptom control as defined by GINA and the control of airway inflammation, measuring quality of life might add useful information, particularly regarding exercise limitations and environmental exposures.

To the best of our knowledge, this is the first study to examine the associations between the definition of current symptom control by the present GINA criteria, quality of life and sputum cellularity. In the present study, nearly seven in 10 subjects with controlled asthma had paucigranulocytic sputum, suggesting that negative responses to the four questions of the present GINA symptom control criteria are good indicators of the control of airway inflammation. In contrast, in subjects with partly controlled or uncontrolled asthma, the presence of symptoms was not an indicator of airway inflammation or

TABLE 2 Mean overall Standardised Asthma Quality of Life Questionnaire scores and scores for each domain according to the Global Initiative for Asthma (GINA) 2010-2012 levels of asthma control ${ }^{\#}$

\section{Controlled asthma (CA)}

43

$6.1(5.7-6.4)$

Overall score

Mean differences

Symptoms domain score

Mean differences

Activity limitation domain score

Mean differences

Emotional function domain score

Mean differences

Environmental stimuli domain score

Mean differences

$6.1(5.7-6.4)$

$5.8(5.3-6.3)$

$5.9(5.4-6.4)$

\section{Partly controlled asthma (PCA)}

37

$5.3(4.9-5.7)$

CA versus $\mathrm{PCA}=0.8(0.2-1.4) ; \mathrm{p}=0.006$

CA versus UA=2.7 (2.1-3.3); $p<0.001$

PCA versus UA=1.9 (1.2-2.5); $p<0.001$

$5.6(5.2-6.0)$

$3.8(3.4-4.1)$

$<0.001$

CA versus $P C A=0.6(0.1-1.2) ; p=0.03$

CA versus UA=2.5 (2.1-3.4); $p<0.001$

$P C A$ versus $U A=2.1(1.4-2.7) ; p<0.001$

$5.2(4.8-5.6)$

CA versus PCA $=0.9(0.3-1.5) ; p=0.002$

CA versus UA=2.6 (2.0-3.2); $\mathrm{p}<0.001$

PCA versus UA=1.7 (1.0-2.3); $p<0.001$

$5.4(4.8-5.9)$

CA versus PCA $=0.5(-0.3-1.4) ; p=0.3$

$C A$ versus $U A=2.8(1.9-3.6) ; p<0.001$

$P C A$ versus $U A=2.2(1.3-3.1) ; p<0.001$

$4.6(4.1-5.2)$

Uncontrolled asthma (UA)

\section{3}

$3.6(3.2-4.0)$

$<0.001$

CA versus PCA $=1.3(0.4-2.2) ; p=0.002$

$C A$ versus $U A=2.9(1.9-3.8) ; p<0.001$

PCA versus UA=1.6 (0.6-2.5); $p<0.001$

p-value 


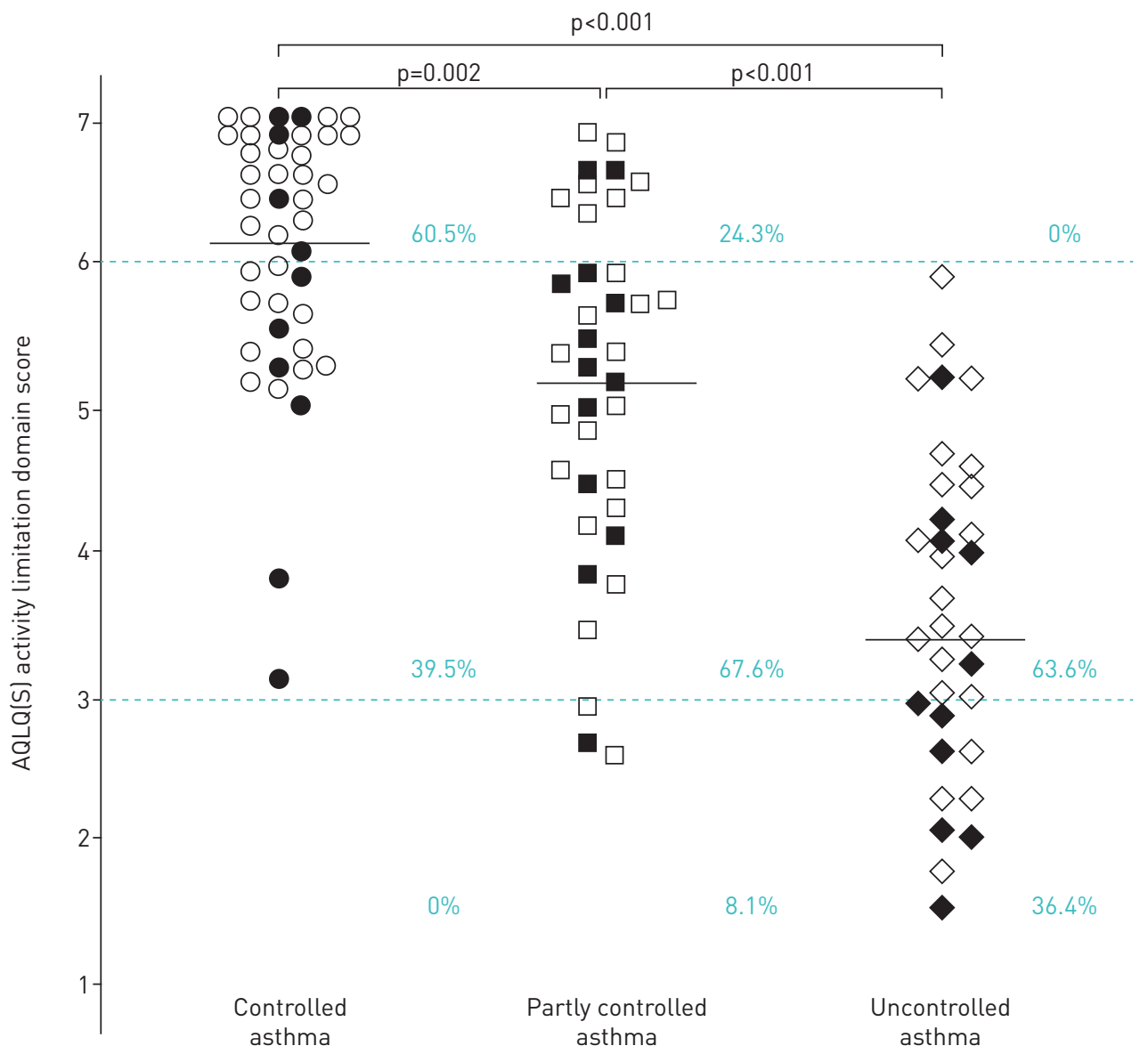

FIGURE 2 Individual plots of Standardised Asthma Quality of Life Questionnaire (AQLQ(S)) scores for the activity limitation domain in each Global Initiative for Asthma level of asthma control (controlled, partly controlled and uncontrolled). Solid symbols: eosinophilic sputum; open symbols: noneosinophilic sputum. The mean of each group is indicated. The upper dashed line represents the lower limit of the AQLQ(S) score ( $\geqslant 6$ ) that indicates minimal or no impairment; the lower dashed line represents the upper limit of the AQLQ(S) score $(<3)$ that indicates severe impairment; the area between the dashed lines represents AQLQ(S) score $\geqslant 3$ and $<6$, indicating moderate/some impairment. Percentage values indicate the proportion of subjects in each level of asthma control with minimal or no impairment, moderate/some impairment, or severe impairment.

eosinophilic inflammation. Accordingly, one-third of subjects with partly controlled or uncontrolled asthma had paucigranulocytic sputum, suggesting that in these scenarios, other underlying physiopathologies or causes may explain the symptoms. Interestingly, the proportion of eosinophilic sputum was similar between asthma control groups $(25.2 \%, 35.1 \%$ and $33.3 \%$ in subjects with controlled, partly controlled and uncontrolled asthma, respectively). This is not unexpected [9], and it is in keeping with a previous report showing that the ability of clinical assessment to predict the presence and type of inflammation is poor [21]. Taken together, these results suggest that in partly controlled and uncontrolled asthma, induced sputum adds relevant information regarding the current level of asthma control, despite the recognised limitation of induced sputum not being easily available in clinical practice.

This is also the first study to demonstrate the impact of asthma in patients with controlled asthma. In the present study, $\sim 40 \%$ subjects with controlled asthma had moderate/some impairment, largely due to activity limitations and environmental stimuli exposure. Although both the present GINA symptom question [2] and the AQLQ(S) [5] assess activity limitations, the AQLQ(S) is more comprehensive as 11 out of 32 questions refer to activity limitation, describing different types and intensity of activity as well as the degree of limitation. Thus, the current way of examining exercise limitations when using the GINA criteria may be imprecise because of the lack of offering a standardised detailed way of what is intended from the question "In the past 4 weeks, has the patient had any activity limitation due to asthma" [2]. Our results also imply that asthma symptoms triggered by environmental stimuli indicate lack of control and therefore should be added to the criteria of control. Overall, these results point to the relevance of including quality of life as another dimension of asthma control to help physicians make decisions 


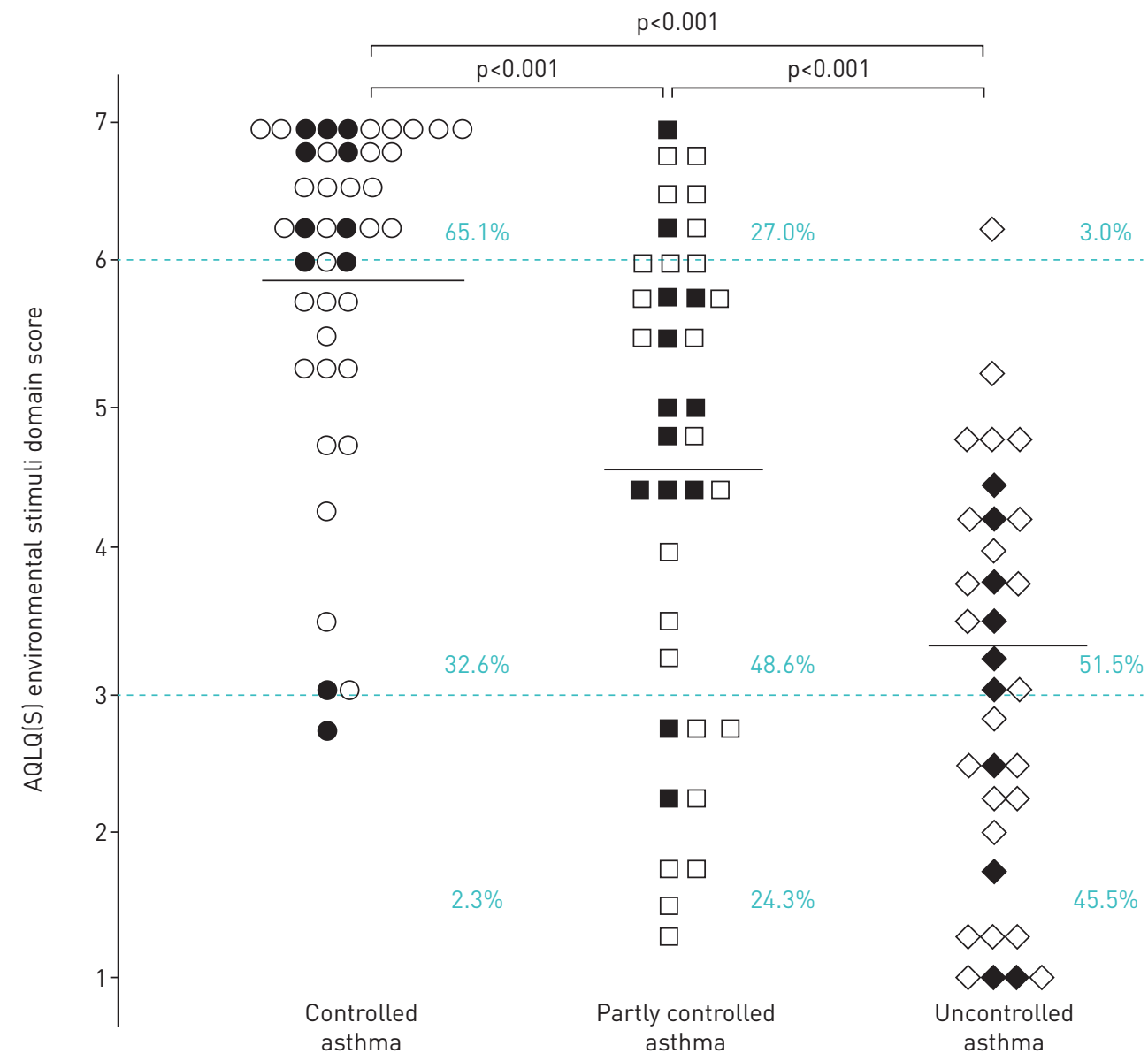

FIGURE 3 Individual plots of Standardised Asthma Quality of Life Questionnaire (AQLQ(S)) scores for the environmental stimuli domain in each Global Initiative for Asthma level of asthma control (controlled, partly controlled and uncontrolled). Solid symbols: eosinophilic sputum; open symbols: noneosinophilic sputum. The mean of each group is indicated. The upper dashed line represents the lower limit of the AQLQ(S) score $(\geqslant 6)$ that indicates minimal or no impairment; the lower dashed line represents the upper limit of the AQLQ(S) score $(<3)$ that indicates severe impairment; the area between the dashed lines represents AQLQ(S) score $\geqslant 3$ and $<6$, indicating moderate/some impairment. Percentage values indicate the proportion of subjects in each level of asthma control with minimal or no impairment, moderate/some impairment, or severe impairment.

regarding changes in treatment, particularly in relation to patients with controlled or partly controlled asthma. This information might be clinically relevant because in order to be able to make clinical decisions at the point of care, the treating physician needs tools that clearly indicate the differences between the levels of control in a given patient.

Accordingly, discerning between controlled asthma and uncontrolled asthma is easily done in clinical practice, leading the treating physician to step-down treatment in the former and step-up treatment in the latter. In contrast, the difference between controlled and partly controlled asthma according to the present GINA criteria [4] may not be so easily determined in a clinical setting, despite being objective. New parameters that help identify potential impairments in partly controlled asthma or even in controlled asthma could be an asset to help guide decisions on asthma treatment. However, the applicability of using quality of life in this context needs to be further investigated in longitudinal studies, as the present study is limited by its cross-sectional nature.

In addition, our results differed from those of previous studies $[9,18]$ by showing that patients with partly controlled asthma have worse quality of life than those with controlled asthma since the impact on quality of life with partly controlled asthma varies from some to severe impairment. In particular, measuring quality of life shows that the majority of asthmatic subjects with partly controlled disease have moderate impairment. Differences in the definition of control and study populations may account for these discrepancies; however, despite these differences, our findings are important because the decision to change asthma treatment in patients with partly controlled asthma may be better implemented knowing 


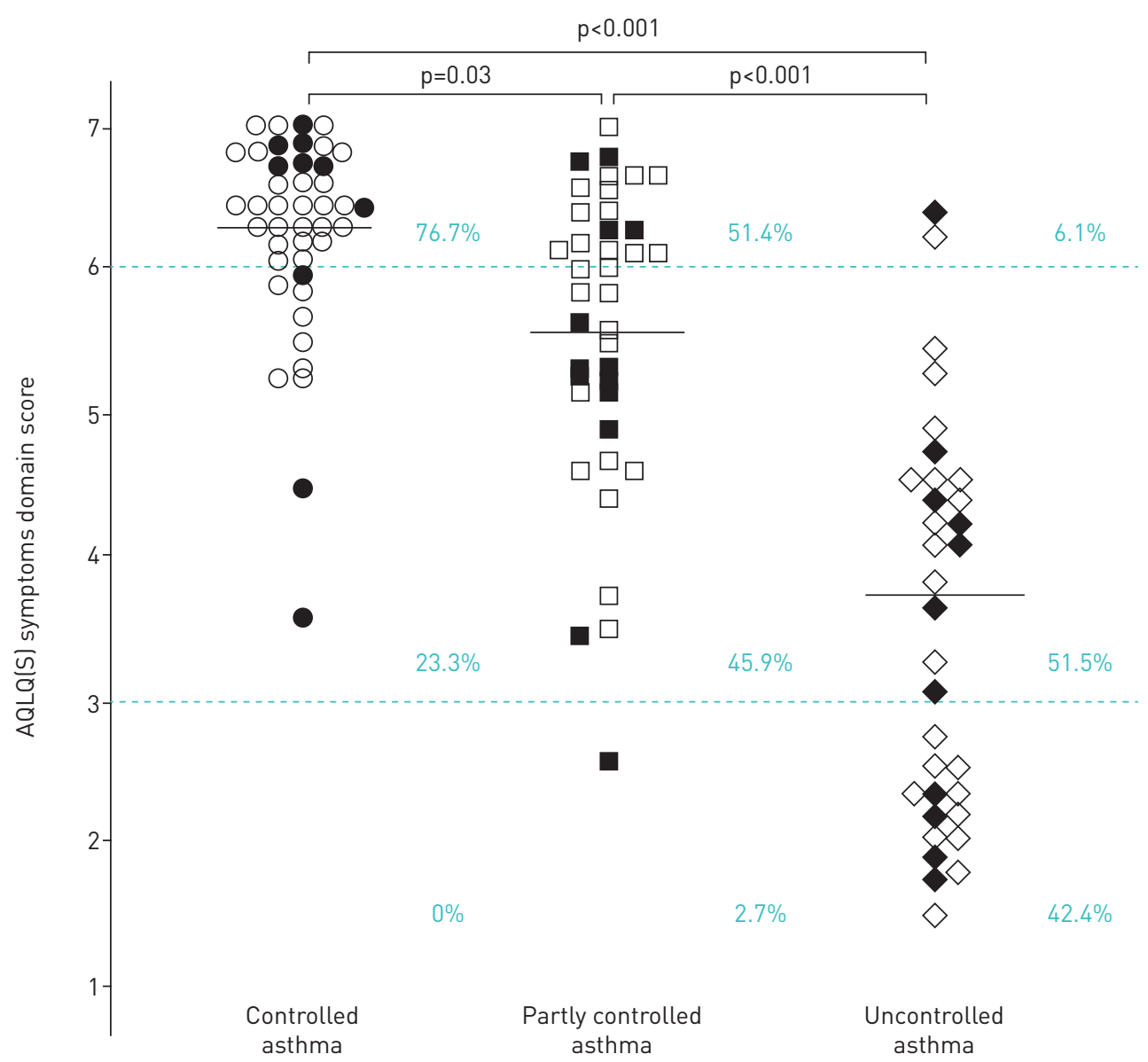

FIGURE 4 Individual plots of Standardised Asthma Quality of Life Questionnaire (AQLQ(S)) scores for the symptoms domain in each Global Initiative for Asthma level of asthma control (controlled, partly controlled and uncontrolled). Solid symbols: eosinophilic sputum; open symbols: noneosinophilic sputum. The mean of each group is indicated. The upper dashed line represents the lower limit of the AQLQ(S) score $(\geqslant 6)$ that indicates minimal or no impairment; the lower dashed line represents the upper limit of the AQLQ(S) score $(<3)$ that indicates severe impairment; the area between the dashed lines represents AQLQ(S) score $\geqslant 3$ and $<6$, indicating moderate/some impairment. Percentage values indicate the proportion of subjects in each level of asthma control with minimal or no impairment, moderate/some impairment, or severe impairment.

the impact on quality of life. Nevertheless, these results need to be confirmed by longitudinal interventional studies.

The correlation between asthma control and quality of life has been previously investigated using various validated control questionnaires or composites [9], but not in relation to GINA current symptom control [4]. Consistent with previous reports, our results showed that the better the control is, the better the quality of life will be. However, in contrast to previous studies $[9,18]$, our results have shown a significant and clinically important difference between controlled asthma and partly controlled asthma, which may be due to differences in the study population and/or the definition of asthma control.

In the present study, the definition of current control did not include lung function, although this does not seem to be the reason for the differences in quality of life between controlled asthma and partly controlled asthma. When stricter criteria were used as the definition of current control by a former edition of GINA guidelines (which included spirometry on treatment) [22], the magnitude of the differences remained similar (data not shown). There are limitations in the present study. The cross-sectional design does not allow for the establishment of the longitudinal stability of the results. Additional longitudinal and interventional studies are required to address this issue. Therefore, our results should be interpreted with caution.

In conclusion, we have shown that the present GINA definition of current symptom control in the majority of patients is associated with paucigranulocytic sputum and better quality of life, suggesting that the GINA definition of control reflects control of airway inflammation. However, when asthma is partly controlled or uncontrolled according to the GINA criteria, airway inflammation may be eosinophilic, neutrophilic or 
absent and in this situation measuring airway inflammation may be beneficial. In addition, the results suggest that including quality of life as another dimension of asthma control may add useful information.

Acknowledgements: We thank Celia Zimmermann and Jessica Gonçalves (NUPAIVA Research Center, Federal University of Santa Catarina, Florianópolis, Brazil) for processing the induced sputum and managing quality control.

Author contributions: C.C. Rocha, M.M.M. Pizzichini and E. Pizzichini developed the study protocol. All authors critically revised the manuscript with important intellectual contributions. All authors approved the final version.

Conflict of interest: M.M.M. Pizzichini has nothing to disclose. C.C. Rocha has nothing to disclose. M.G. de Souza Tavares has nothing to disclose. L.J.M. Steidle has nothing to disclose. R. Maureci da Silva has nothing to disclose. F. dal Pizzol has nothing to disclose. P.G. Gibson reports receiving grants and personal fees from AstraZeneca, GSK and Novartis, outside the submitted work. E. Pizzichini has nothing to disclose.

Support statement: This study was supported by the NUPAIVA Research Center and CAPES (Coordenação de Aperfeiçoamento de Pessoal de Nível Superior), Brazil.

\section{References}

1 Reddel HK, Taylor DR, Bateman ED, et al. An official American Thoracic Society/European Respiratory Society statement: asthma control and exacerbations: standardizing endpoints for clinical asthma trials and clinical practice. Am J Respir Crit Care Med 2009; 180: 59-99.

2 Global Initiative for Asthma. Global Strategy for Asthma Management and Prevention. 2018. https://ginasthma. org/gina-reports/ Date last accessed: December 22, 2018.

3 Fu JJ, McDonald VM, Wang G, et al. Asthma control: how it can be best assessed? Curr Opin Pulm Med 2014; 20 $1-7$.

4 Global Initiative for Asthma. Global Strategy for Asthma Management and Prevention. 2014. https://ginasthma. org/archived-reports Date last accessed: December 22, 2018.

5 Juniper EF, O’Byrne PM, Guyatt GH, et al. Development and validation of a questionnaire to measure asthma control. Eur Respir J 1999; 14: 902-907.

6 Nathan RA, Sorkness CA, Kosinski M, et al. Development of the asthma control test: a survey for assessing asthma control. J Allergy Clin Immunol 2004; 113: 59-65.

7 Skinner EA, Diette GB, Algatt-Bergstrom PJ, et al. The Asthma Therapy Assessment Questionnaire (ATAQ) for children and adolescents. Dis Manag 2004; 7: 305-313.

8 Reddel HK, Bateman ED, Becker A, et al. A summary of the new GINA strategy: a roadmap to asthma control. Eur Respir J 2015; 46: 622-639.

9 Volbeda F, Broekema M, Lodewijk ME, et al. Clinical control of asthma associates with measures of airway inflammation. Thorax 2013; 68: 19-24.

10 Bateman ED, Boushey HA, Bousquet J, et al. Can guideline-defined asthma control be achieved? The Gaining Optimal Asthma ControL Study. Am J Respir Crit Care Med 2004; 170: 836-844.

11 Juniper EF, Buist AS, Cox FM, et al. Validation of a standardized version of the Asthma Quality of Life Questionnaire. Chest 1999; 115: 1265-1270.

12 Pepys J. Skin test in diagnosis. In: Gell PGH, Coombs RRA, Lachmann PJ, eds. Clinical Aspects of Immunology. 3rd Edn. Oxford, Blackwell Scientific, 1975; pp. 55-80.

13 American Thoracic Society. Standardization of spirometry, 1994 update. Am J Respir Crit Care Med 1995; 152 $1107-1136$.

14 Crapo RO, Morris AH, Gardner RM. Reference spirometric values using techniques and equipment that meet ATS recommendations. Am Rev Respir Dis 1981; 123: 659-664.

15 Juniper EF, Cockcroft DW, Hargreave FE. Histamine and Methacholine Inhalation Test: A Laboratory Tidal Breathing Protocol. Lund, Astra, 1994.

16 Juniper EF. Measurement of Health-Related Quality of Life \& Asthma Control. www.qoltech.co.uk/languages.htm Date last accessed: December 22, 2018.

17 Juniper EF, Guyatt GH, Willan A, et al. Determining a minimal important change in a disease-specific Quality of Life Questionnaire. J Clin Epidemiol 1994; 47: 81-87.

18 Bateman ED, Bousquet J, Keech ML, et al. The correlation between asthma control and health status: the GOAL study. Eur Respir J 2007; 29: 56-62.

19 Pizzichini E, Pizzichini MM, Efthimiadis A, et al. Indices of airway inflammation in induced sputum: reproducibility and validity of cell and fluid-phase measurements. Am J Respir Crit Care Med 1996; 154: 308-317.

20 Wang F, He XY, Baines KJ, et al. Different inflammatory phenotypes in adults and children with acute asthma. Eur Respir J 2011; 38: 567-574.

21 Parameswaran K, Pizzichini E, Pizzichini MM, et al. Clinical judgement of airway inflammation versus sputum cell counts in patients with asthma. Eur Respir J 2000; 15: 486-490.

22 Global Initiative for Asthma. Global Strategy for Asthma Management and Prevention. 2012. https://ginasthma. org/archived-reports Date last accessed: December 22, 2018. 